\title{
Device Physics and Simulation of Metal/Ferroelectric-Film/p-type Silicon Capacitors
}

Hisham Z. Massoud*

Max-Planck Institute of Microstructure Physics, Weinberg 2, D-06120 Halle/Saale, Germany.

This paper introduces an electrostatic model for Metal/Ferroelectric/Silicon (MFS) capacitors. These structures consist of a metal gate, a dielectric stack which includes a ferroelectric film, and a $p$-type silicon substrate. The dielectric stack consists of a switching ferroelectric layer and two nonswitching dielectric or buffer layers. This model predicts the dependence of the polarization charge density $\mathcal{P}_{d}$, the electric field in the ferrolelectric film $\mathcal{E}_{f_{e}}$, the voltage across the dielectric stack $V_{O X}$, the semiconductor surface potential $\psi_{S C}$, and the semiconductor charge density $Q_{S C}$ on the gate-to-bulk voltage $V_{G B}$ under static conditions. The low- and high-frequency capacitance-voltage characteristics of MFS capacitors are calculated.

\section{INTRODUCTION}

Studies of ferroelectric capacitors [1] and ferroelectric nonvolatile-memory field-effect transistors [2] have focused mainly on the analysis of the behavior of these devices in the SawyerTower circuit which is commonly used to measure their ferroelectric properties, and in circuits with asymmetric nonperiodic input signals with arbitrary intitial conditions [3]. In this paper, however, we model the device physics of ferroelectric capacitors fabricated on $p$-type silicon substrates using a simple electrostatic model, with the goal of predicting their lowand high-frequency capacitance-voltage characteristics, and relate their $C_{g b}\left(V_{G B}\right)$ hysterisis to the properties of the ferroelectric film.

\section{MFS DEVICE MODEL}

The metal-ferroelectric-silicon (MFS) capacitor modeled in this paper is similar to that described in [3] and is shown in Fig. 1. The dielectric stack consists of three layers. Buffer layers 1 and 2

\footnotetext{
* Permanent Address: Semiconductor Research Laboratory, Department of Electrical and Computer Engineering, Duke University, Durham, NC 27708-0291, U.S.A. The author acknowledges the support of U. M. Gösele and many helpful discussions with M. Alexe and A. Pignolet.
}

are two non-dipole-switching dielectric layers of thicknesses $X_{1}$ and $X_{2}$ and dielectric permittivities $\epsilon_{1}$ and $\epsilon_{2}$, respectively. The ferroelectric film contained between the buffer layers is of thickness $X_{f e}$ and linear dielectric permittivity $\epsilon_{f e}$.

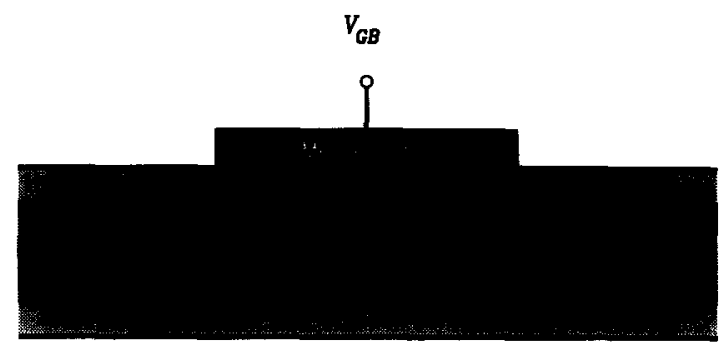

$p$-type Silicon Substrate

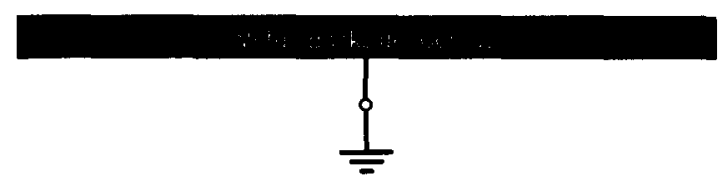

Fig. 1. Metal-Oxide-Ferroelectric-Oxide-p(Si) (MFS) capacitor modeled in this work.

The gate-to-bulk voltage $V_{G B}$ generates a field 
$\mathcal{E}_{f e}$ in the ferroelectric film that induces a dielectric polarization charge density $\mathcal{P}_{d}$. The dipole polarization $\mathcal{P}_{d}$ depends on $\mathcal{E}_{f e}$ and on the history of the field applied across the material. After several cycles of voltages applied in both polarities, the polarization reaches its saturated state. The dependence of the saturated $\mathcal{P}_{d}$ on $\mathcal{E}_{f e}$ is shown in Fig. 2.

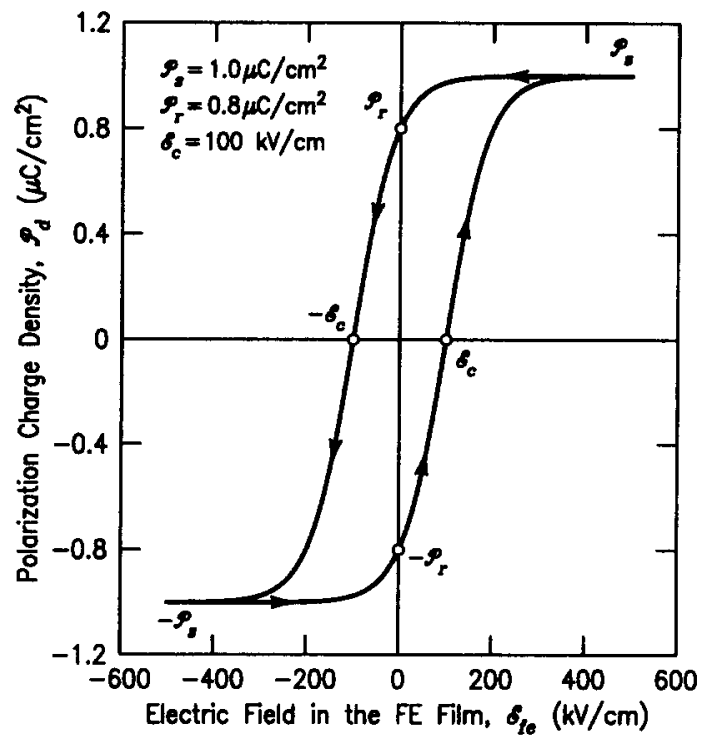

Fig. 2. Dependence of dielectric polarization $\mathcal{P}_{d}$ on the electric field $\mathcal{E}_{f e}$ in a MFS capacitor.

The saturated polarization loop is defined by

$\mathcal{P}_{d}^{+}\left(\mathcal{E}_{f e}\right)=\mathcal{P}_{s} \tanh \left(\left(\mathcal{E}_{f e}-\mathcal{E}_{\mathrm{c}}\right) / 2 \delta\right)$,

$\mathcal{P}_{d}^{-}\left(\mathcal{E}_{f e}\right)=-\mathcal{P}_{d}^{+}\left(-\mathcal{E}_{f e}\right)$,

where

$$
\delta=\frac{\mathcal{E}_{c}}{\ln \left(\left(1+\mathcal{P}_{r} / \mathcal{P}_{s}\right) /\left(1-\mathcal{P}_{r} / \mathcal{P}_{s}\right)\right)},
$$

where $\mathcal{P}_{r}$ is the remnant polarization, $\mathcal{P}_{\text {s }}$ the spontaneous polarization, and $\mathcal{E}_{c}$ the coercive field. The electrostatic potential distribution in the device is written as

$V_{G B}=\phi_{M S}+V_{O X}+\psi_{S C}$,

where $\phi_{M S}$ is the workfunction difference between the metal gate and the silicon substrate, $V_{O X}=V_{1}+V_{f e}+V_{2}$ the voltage across the dielectric stack, and $\psi_{S C}$ the electrostatic potential across the silicon space-charge region. We assume that the oxide fixed charge density $Q_{f}$ and the interface-trap charge density $Q_{i t}$ exist at the $\mathrm{Si}-\mathrm{SiO}_{2}$ interface. The positive $x$ direction is taken as going into the silicon from the $\mathrm{Si}-\mathrm{SiO}_{2}$ interface with $x=0$ being at the interface. The charge density on the gate electrode is $Q_{G}$ and that in the semiconductor spage-charge region is $Q_{S C}$. When the electric field is in the positive $x$ direction, the polarization charge density is $\mathcal{P}_{d}$ at $x=-X_{2}$, and $-\mathcal{P}_{d}$ at $x=-\left(X_{f e}+X_{2}\right)$. The charge conservation is written as

$Q_{G}+Q_{i t}+Q_{f}+Q_{S C}=0$.

Applying Gauss's law at all interfaces, we relate the electric field intensity in the dielectric films, the ferroelectric film and in the silicon substrate at the $\mathrm{Si}-\mathrm{SiO}_{2}$ interface to the charge densities. The relationship between the field at the silicon surface and $Q_{S C}$ is the same as that in MOS devices [4]. By combining these results, we find that

$V_{G B}=\phi_{M S}+\frac{Q_{G}}{C_{d s}}-\frac{X_{f e}}{\epsilon_{f e}} \mathcal{P}_{d}+\psi_{S C}$,

where the dielectric-stack capacitance $C_{d s}$ is

$C_{d s}=\left(X_{1} / \epsilon_{1}+X_{f e} / \epsilon_{f e}+X_{2} / \epsilon_{2}\right)^{-1}$,

and the gate-to-bulk capacitance is given by

$C_{g b}=\left(C_{1}^{-1}+C_{f e}^{-1}+C_{2}^{-1}+C_{s c}^{-1}\right)^{-1}$,

where $C_{f e}=\left(\epsilon_{f e}+d \mathcal{P}_{d} / d \mathcal{E}_{f e}\right) / X_{f e}, C_{1}=\epsilon_{1} / X_{1}$ and $C_{2}=\epsilon_{2} / X_{2}$.

In simulating the behavior of MFS structures, we start by assuming the value of $\mathcal{E}_{f e}$. We then calculate $\mathcal{P}_{d}$ from Eqs.(1)-(3) taking into account whether $\mathcal{E}_{f e}$ is increasing or decreasing. Using Gauss's law at the two interfaces of the ferroelectric film, we obtain the electric fields in the two buffer layers. Repeating this at the gate/bufferlayer interface we obtain $Q_{G}$, and at the $\mathrm{Si}-\mathrm{SiO}_{2}$ interface we obtain the electric field in the silicon at $x=0$. This then allows us to calculate $\psi_{S C}$. Using Eq. (6), we obtain $V_{G B}$. In the results, it is seen that all the device electrical parameters reflect the polarization hysterisis. It was also assumed that the current conduction in the films was small enough to be neglected, and that no carrier trapping takes place in anyone of the dielectric films. The results of the simulations are shown as functions of the terminal voltage $V_{G B}$. 


\section{SIMULATION RESULTS}

Simulation results were obtained for MFS capacitors built on $p$-type silicon substrates with $N_{A}=4.0 \times 10^{15} \mathrm{~cm}^{-3}, \mathcal{P}_{s}=1.0 \mu \mathrm{C} / \mathrm{cm}^{2}$, $\mathcal{P}_{r}=0.8 \mu \mathrm{C} / \mathrm{cm}^{2}, \mathcal{E}_{c}=100 \mathrm{kV} / \mathrm{cm}, X_{1}=300 \AA$, $X_{2}=10 \AA, X_{f e}=1000 \AA, \epsilon_{f e}=10 \epsilon_{0} \mathrm{~F} / \mathrm{cm}$, $\epsilon_{1}=\epsilon_{2}=3.9 \epsilon_{0}$ and $\phi_{M S}=-1.1 \mathrm{~V}$. These results are shown in Figs. 3 to 7 . The dependence of the electric field in the ferroelectric film $\mathcal{E}_{f e}$ on the gate-to-bulk voltage $V_{G B}$ is shown in Fig. 3 . A strong dependence of the value of $\mathcal{E}_{f e}$ is observed depending on whether $V_{G B}$ is increasing or decreasing. Similarly, it is seen that the MFS capacitor can have two different values of $\mathcal{E}_{f e}$ for the same $V_{G B}$, again depending on whether the field in the dielectric film is increasing or decreasing.

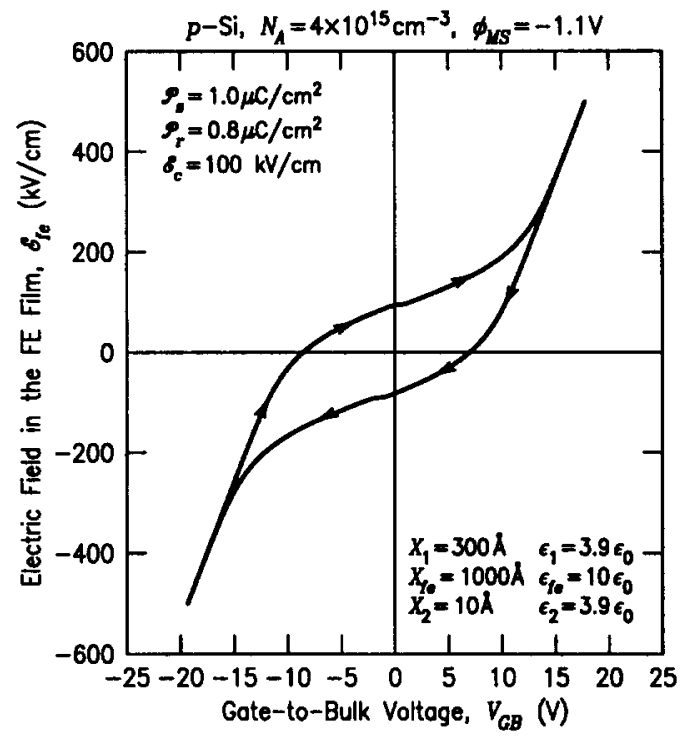

Fig. 3. Dependence of electric field in the ferroelectric film $\mathcal{E}_{f e}$ on the gate-to-bulk voltage $V_{G B}$.

The dependence of the condition in the silicon substrate on the semiconductor potential $\psi_{S C}$, i.e. whether the semiconductor is in accumulation, depletion or inversion, influences the dependence of $\mathcal{E}_{f e}$ on $V_{G B}$. This influence is even more pronounced in the dependence of $\mathcal{P}_{d}$ on $V_{G B}$ as seen in Fig. 4. It is also clear from Figs. 3-8 that the symmetry of the behavior in any of the pa- rameters with $V_{G B}$ is not present as a direct result of the nonzero workfunction difference.

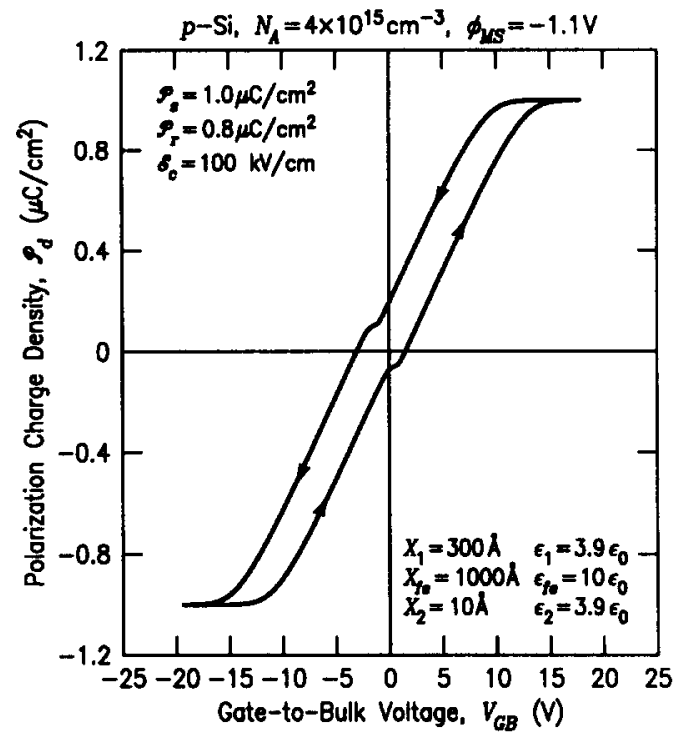

Fig. 4. Dependence of dielectric polarization $\mathcal{P}_{d}$ on the gate-to-bulk voltage $V_{G B}$.

The small kinks observed in Figs. 3 and 4 are the points at which the semiconductor is depleted. It is clear from these two figures that the gateto-bulk voltage at which the semiconductor goes from accumulation to depletion or from depletion to inversion, or vice versa, depends on the direction of the electric field in the ferroelectric film. This observation is easily illustrated in the dependence of the semiconductor potential $\psi_{S C}$ on $V_{G B}$ shown in Fig. 5 . If we consider the points at which $\psi_{S C}=0$, i.e. the depletion to accumulation or accumulation to depletion points, we find that these occur at two different values of $V_{G B}$.

The gate-to-bulk capacitance $C_{g b}$ of the MFS capacitor structure was calculated using Eq. (8), assuming that the frequency dependence is only determined to the response of the semiconductor substrate. The low- and high-frequency capacitances are shown in Figs. 6 and 7, respectively. The low-frequency capacitance of the semiconductor was calculated using the analytical expression for $C_{s c}$ in [4]. The high-frequency capacitance of the semiconductor was obtained by numerically determining the width $W_{D E P}$ of the de- 
pletion region and calculating the high-frequency $C_{s c}$ as $\epsilon_{S i} / W_{D E P}$.

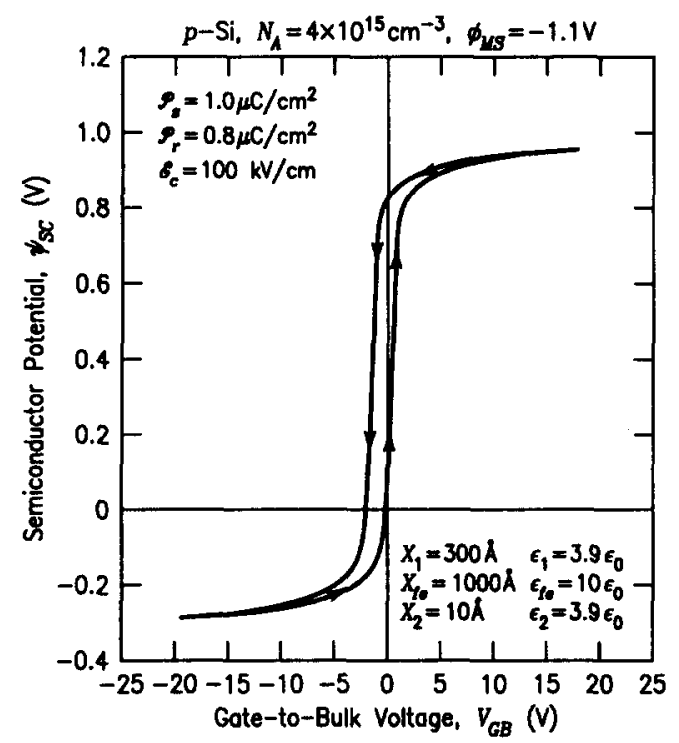

Fig. 5. Dependence of the semiconductor potential $\psi_{S C}$ on the gate-to-bulk voltage $V_{G B}$.

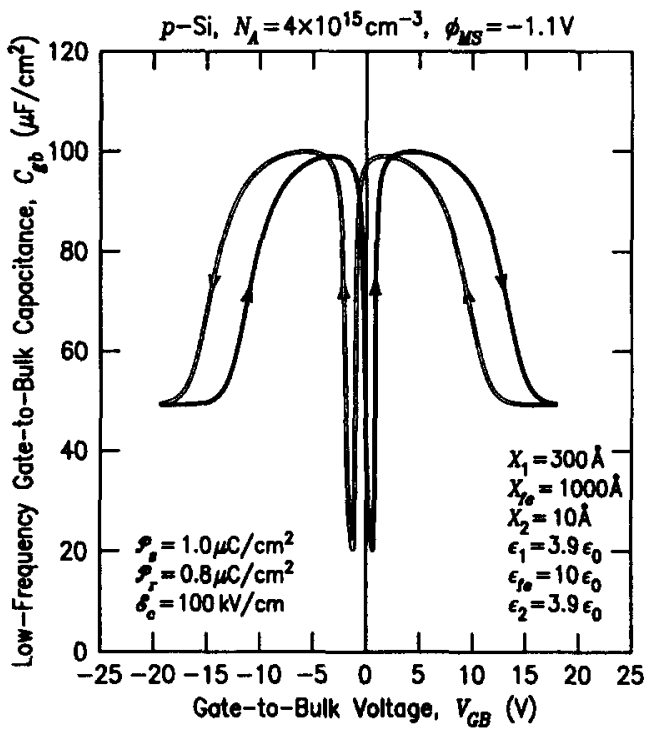

Fig. 6. Dependence of the low-frequency capacitance of a MFS capacitor on the gate-to-bulk voltage $V_{G B}$.

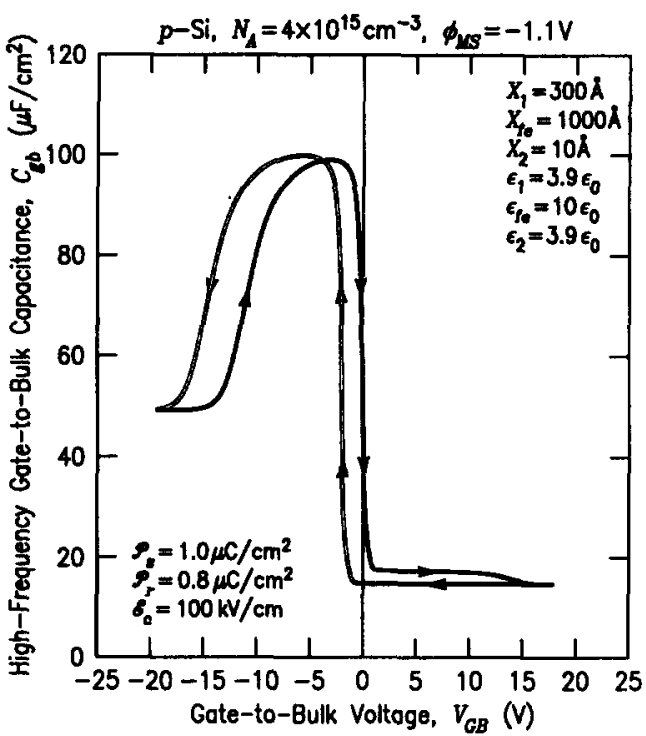

Fig. 7. Dependence of the high-frequency capacitance of a MFS capacitor on the gate-to-bulk voltage $V_{G B}$.

\section{CONCLUSIONS}

The electrostatic model of MFS structures introduced in this paper can predict the dependence of all the electrical variables in the structure on the applied voltage and the electric field in the ferroelectric film. It allows us to define the flatband and threshold voltages in a MFS structure and predict the dependence of the hysterisis in the capacitance-voltage characteristics on the physical parameters of the structure [5].

\section{REFERENCES}

1. S. L. Miller, R. D. Nasby, J. R. Schwank, M. S. Rodgers, and P. V. Dressendorfer, J. Appl. Phys., 68, 6463 (1990).

2. S. L. Miller and P. J. McWhorter, J. Appl. Phys., 72, 5999 (1992).

3. S. L. Miller, J. R. Schwank, R. D. Nasby, and M. S. Rodgers, J. Appl. Phys., 70, 2849 (1991).

4. S. M. Sze, Physics of Semiconductor Devices, John Wiley, 1981.

5. H. Z. Massoud, unpublished results. 